\title{
La enseñanza de las Relaciones Laborales en España: de la homogeneidad a la diversidad
}

\author{
Aglistin Galán Garcia *
}

Desde hace algunos años la Universidad española viene conociendo un proceso de transformación importante, motivado fundamentalmente por los cambios de planes de estudios que se están produciendo. En el caso que nos ocupa, la antigua diplomatura de Graduado Social ha dejado paso a la nueva titulación de diplomado en Relaciones Laborales.

Hasta el 4 de enero de 1996 se habían aprobado en el Consejo de Universidades un total de 42 nuevos planes de estudios para la Diplomatura de Relaciones Laborales. Desde el 31 de julio de 1991, en que se aprobaron los de la Universidad Carlos III de Madrid y los de la Universidad Jaume I de Castellon, hasta hoy, ha habido tiempo, más que suficiente para conocer las principales dificultades que plantean la puesta en práctica de los mencionados planes. Del mismo modo, también ha transcurrido el tiempo necesario como para proponer e intentar determinadas soluciones. Fueron precisamente la evidencia de aquellas dificultades y, entre ellas, la diversidad en los contenidos de las asignaturas que se venían impartiendo, lo que nos movió a convocar en la Universidad de Huelva, las Primeras Jornadas Nacionales sobre la enseñanza en las Relaciones Laborales.

Con dicho motivo tuvimos la oportunidad de reflexionar ampliamente sobre la cuestión. Pero antes de entrar a explicar su desarrollo y las conclusiones alcanzadas, me gustaría hacer una serie de observaciones de carácter general:

1.- La precipitación con que en la mayor parte de los casos se han elaborado y se han puesto en marchalos diversos planes de estudio, ha condicionado en gran medida algunas de las dificultades que iremos comentando.

2.- Respecto de los contenidos, se ha producido un cambio radical en el panorama académico de los diplomados, pasándose de una total homogede Huelva.

* Agustín Galán García es Director de la Escuela Universitaria de Relaciones Laborales 
neidad a una gran diversidad. El plan de estudios de 1980 se promulgó con un carácter común para todas las Escuelas de Graduados Sociales, sin dejar espacio alguno para que cada centro estableciera asignaturas o materias particulares. El grado de uniformidad que establecía en cuanto al contenido de la docencia era, por ello, completo. Durante su vigencia existió una total homogeneidad en la formación que recibían los alumnos de la diplomatura de Graduado Social.

La uniformidad que se pretendía no operaba exclusivamente respecto de los centros que impartían esta titulación, sino que se extendía al propio alumnado, al que no se dejaba opción alguna en cuanto a las asignaturas a cursar. No se preveían así, asignaturas optativas o cualquier otro procedimiento por el que el estudiante pudiera seleccionar y formar su propio itinerario curricular.

El plan presentaba una carga lectiva de 180 créditos, distribuidos en tres cursos académicos homogéneos de 60 cada uno. La base para la ordenación del curso era un modelo homogeneo de asignatura anual, al que se adaptaban la mayoría de las materias objeto de atención en el plan. Junto a este modelo, y con un carácter marcadamente complementario, existían algunas asignaturas cuatrimestrales, dos por curso académico, que se sucedían en el tiempo, de tal modo que en todo momento la carga lectiva era la misma.

La homogeneidad a la que nos venimos refiriendo alcanzaba también al perfil profesional del Diplomado que la cursara. Se trataba de formar a "Técnicos en materias sociales y laborales".

De aquella situación de homogeneidad consolidada, se ha pasado al polo opuesto, a una gran diversidad, excesiva diría yo, amparada en la L.R.U. y que posibilita a las distintas universidades -respetando la troncalidad existente en el R.D. 1429/1990 (26 de octubre)- a formar su propio plan de estudios.

Algunos datos para explicar la situación planteada: 
Cuadro n⿳0 1: Carga Lectiva, Créditos asignados a materias troncales y obligatorias ${ }^{1}$

\begin{tabular}{||c|c|c|c||}
\hline \hline & $\begin{array}{c}\text { Carga lectiva } \\
\text { total }\end{array}$ & $\begin{array}{c}\text { Créditos materias } \\
\text { troncales }\end{array}$ & $\begin{array}{c}\text { Créditos materias } \\
\text { obligatorias }\end{array}$ \\
\hline Media & 209.29 & 128 & 36.39 \\
\hline Mediana & 207 & 124 & 38 \\
\hline Máximo & $264(1)$ & 162 & $85(5)$ \\
\hline Mínimo & $180(2)$ & $102(4)$ & \\
\hline
\end{tabular}

(1) U. Vigo (2) U. Pompeu Fabra y U. Granada (Melilla) (3) U. Castilla La Mancha (4) U.Carlos III (5) U. Vigo

Cuadro $n^{\circ}$ 2: Créditos asignados a optativas y libre configuración

\begin{tabular}{||c|c|c|c||}
\hline \hline & Créditos optativas & $\begin{array}{c}\text { Oferta Total } \\
\text { Optativas }\end{array}$ & $\begin{array}{c}\text { Créditos Libre } \\
\text { Configuración }\end{array}$ \\
\hline Media & 21.19 & 68.39 & 20.40 \\
\hline Mediana & 21 & 66 & 21 \\
\hline Máximo & $52(1)$ & $196(3)$ & $28(5)$ \\
\hline Mínimo & $0(2)$ & $0(4)$ & $12(6)$ \\
\hline
\end{tabular}

(1) U.Valencia (2) U. Islas Baleares (3) U. Valencia (4) U. Islas Baleares (5) U. Carlos III (6) U. Castilla La Mancha

'RODRÍGUEZ-PIÑERO ROYO, M. Y UGALDE GONZÁLEZ, I.: "Situación actual de los planes de estudio de la Diplomatura en Relaciones Laborales: la ampliación de estudios (Segundo Ciclo)". Ponencia presentada en el X Congreso Nacional de Juntas de Gobierno de los Colegios de Graduados Sociales y Diplomados en Relaciones Laborales. Las Palmas de Gran Canaria. Mayo. 1995. (en prensa). 
Cuadro nº 3: Cárga lectiva total, créditos teóricos y prácticos

\begin{tabular}{||c|c|c|c||}
\hline & $\begin{array}{c}\text { Carga lectiva } \\
\text { total }\end{array}$ & Crédito teóricos & Créditos prácticos \\
\hline Media & 209.29 & 118.35 & 78.99 \\
\hline Mediana & 207 & 118.5 & 75 \\
\hline Máximo & 264 & 143 & 120 \\
\hline Mínimo & 180 & 95 & 51 \\
\hline
\end{tabular}

Este cambio tan radical y tan repentino ha provocado situaciones de inseguridad tanto en la docencia como en la gestión, que se han tenido que ir solucionando sobre la marcha, con el agravante de que en muchas ocasiones, y debido precisamente a la mencionada diversidad, la experiencia de unos no era asumible por otros.

Fue la heterogeneidad existente en el contenido de un buen número de asignaturas e incluso de asignaturas troncales, lo que nos llevó a considerar la conveniencia de convocar unas jornadas dirigidas a docentes que contaran con experiencia en las Escuelas de Relaciones Laborales. El planteamiento de las jornadas iba a obedecer a una doble motivación; por un lado, averiguar qué se estaba explicando a los alumnos en dichas escuelas y, en segundo lugar, intentar unificar los contenidos, o al menos establecer los cauces para ello. Al mismo tiempo, se trataba de responder a una pregunta que, a priori podría parecer ciertamente elemental pero que en la práctica, contrastando programas no lo iba a ser tanto. La pregunta era: ¿qué debe aportar cada una de las areas de conocimiento al perfil de un diplomado en Relaciones Laborales?. Se trataba en definitiva, de realizar un esfuerzo de puesta en común, una búsqueda de un marco conceptual común que garantizara la homogeneidad necesaria desde la perspectiva específica de nuestra diplomatura, añadiéndole un profundo sentido autocrítico que reflejara la realidad del proceso seguido desde la puesta en práctica de los nuevos planes de estudio. Eramos conscientes en el momento de iniciar la convocatoria, de que la raiz de la diversidad a la que nos hemos referido se encontraba, en gran medida, en que no todas las escuelas imparten la misma asignatura las mis- 
mas áreas de conocimiento, la relación de fuerzas no es la misma en todas las escuelas, etc.. No obstante, nuestro objetivo pretendía salvar este obtáculo e ir más allá; quería llegar al acuerdo entre docentes, preocupados por la formación de un perfil profesional adecuado a las nuevas necesidades del mercado.

Superada la fase preparatoria nos dispusimos a invitar como ponentes a especialistas de las áreas seleccionadas, en los que se diera además la circunstancia de contar con experiencia docente y, en el mejor de los casos, también con experiencia en la gestión de la nueva diplomatura. Esta circustancia se daba y con creces en el Profesor Faustino Miguélez, para el área de Sociología, en el Profesor Arenas Posadas, para el área de Historia, en el Profesor Sempere Navarro, para el área de Derecho del Trabajo y de la Seguridad Social, en el Profesor Fernández Guerrero para el área de Organización de Empresas y en la Profosera De Paz Bañez, para el área de Economía. En torno a las ponencias que ellos mismos elaboraron se discutieron más de un centenar de comunicaciones.

Con el contexto ya dibujado y para entrar con cierto detalle en el análisis de las dificultades analizadas, siempre desde el punto de vista de la docencia, se dedicó especial atención a las siguientes cuestiones".

a. Solapamiento de asignaturas, que hacen imprescindible una estrechísima coordinación, no sólo en el interior de las áreas que imparten varios programas, sino también entre las distintas áreas de conocimiento que participan en la docencia.

No podemos olvidar en este sentido, la multidisciplinariedad que caracteriza a esta diplomatura y que viene a responder a la complejidad y a la nueva profesión que se está formando en torno a la modernización de las empresas, de las organizaciones y de la administración, en particular de los recursos humanos. Dicha pluridisciplinariedad, siempre que esté bien coordinada, puede proporcionar al alumno una visión muy completa del conjunto de las relaciones laborales, pero si no es así, puede llevarles a una tremenda dispersión en la que resulta muy fácil perderse, y tener dificultades para definir su función social y productiva, así como la falta de un encuadramiento de la actividad profesional propia en relación con las otras a las que habilitan los estudios antes referidos. Esta misma situación, obliga a mantener continuos

\footnotetext{
${ }^{2}$ Los problemas suscitados en la gestión de dichos planes fueron también analizados.
} 
contactos a nivel nacional; de manera institucionalizada si fuera preciso. La discusión sobre programas concretos, metodología docente, intercambio de experiencias, de dificultades, de posibles interpretaciones y soluciones, resulta altamente positivo y necesario al mismo tiempo. De hecho, algunas áreas de las allí reunidas han establecido ya los mecanismos necesarios para permutarse programas, intentar establecer unos contenidos mínimos comunes, una determinda periodización, etc. En este mismo sentido, los resultados que se obtengan dependerán del conjunto de las áreas implicadas. El que algunas de ellas pretendan mantener el protagonismo sería muy perjudicial para el conjunto y para la formación del nuevo profesional.

Una posible solución para evitar los solapamientos mencionados, podría estar en conseguir de los departamentos universitarios una profunda implicación en el desarrollo de esta titulación. Dicha implicación pasaría por procurar que los profesores que impartan docencia en la diplomatura conocieran bien el conjunto del plan de estudios, no únicamente la asignatura o asignaturas que le corresponden impartir. Del mismo modo sería muy útil que conocieran el nuevo perfil del diplomado al que está formando. Caso de producirse, el cambio en la concepción de un buen número de asignaturas sería inmediato. Al mismo tiempo, asignaturas que hoy causan cierto rechazo entre los alumnos por no encontrarles sentido en el conjunto de sus estudios, cobrarían utilidad.

También se podría poner de manifiesto la implicación de los departamentos en nuestra titulación, estimulando el continuo reciclaje del profesorado y la investigación. El éxito de una docencia sólida y dinámica está hoy fuertemente relacionado con la existencia de investigación sobre la problemática que constituye el objeto de la profesión y de la carrera. Es la única manera de captar los cambios que están teniendo lugar, sin perder, por supuesto, la conexión con la reflexión teórica.

b. Programas específicos para la diplomatura. Si hablamos de una nueva titulación, de un nuevo perfil, es necesario adaptar el contenido de los programas a esta nueva orientación. No se pueden explicar en las Escuelas Universitarias de Relaciones Laborales, los mismos programas que se imparten en las licenciaturas de Administración de Empresas, de Economía, Psicología, Sociología, o de Derecho, en la Diplomatura de Epresariales, etc, sencillamente porque esta titulación no es, y no pretende ser ni siquiera parcialmente, Derecho, Sociología, Psicología, Económicas, Administración de Empresas, etc,. Tampoco es una mezcla mas o menos aleatoria de aspectos de 
estas otras titulaciones. De tal manera que la selección de los contenidos debe hacese conforme a un criterio claro de referencia, que no es otro que el estudio y tratamiento de la prestación del trabajo desde sus diversas perspectivas, tanto jurídicas, como organizativas, económicas, psicológicas o sociológicas. Se trata en definitiva, de atribuir un perfil preciso a los estudios, procurando un equilibrio interno de ese enfoque interdisciplinar". Del mismo modo sería deseable introducir en los mencionados programas, el cambio que se aprecia hacia una mayor amplitud de conocimientos entre la diplomatura de Graduado Social y la Diplomatura de Relaciones Laborales.

c. Duración cuatrimestral de las asignaturas. Ya se ha comentado la diferencia con los planes de Graduado Social, donde la mayoria de las asignaturas eran anuales. El cambio de la anualidad al cuatrimestre ha traído como consecuencia una falta de adecuación de los programas al tiempo real disponible para impartirlos. Aquí puede estar una de las causas del alto índice de suspensos en los primeros años de vigencia de los nuevos planes. Ante la tentación de seguir impartiendo los antiguos programas, se hace imprescindible una correcta adecuación de los programas al número de créditos asignados y, por lo tanto, a las horas correspondientes.

d. Concentración del período de exámenes. Con excesiva ligereza se ha achacado el "fracaso" de los nuevos planes a ésta circusntancia. Creo que no es ésta una de las causas mas importantes si tenemos en cuenta que los nuevos planes suponen entrar en una nueva dinámica, en nuevo ritmo de trabajo y un esfuerzo de adaptación para todos. Los antiguos permitían a los alumnos concentrar el período intenso de estudios en los días previos al examen y afrontarlo con sólidas garantías de superarlo. Ahora esto no es posible. Nos exige un esfuerzo continuado a lo largo de todo el cuatrimestre. Si antes se concentraba el período de trabajo en las épocas de exámenes y se dilataban las vacaciones durante el resto del curso, ahora se dilata el período de trabajo y se concentran las vacaciones

e. Por último y a modo de conclusión para este apartado, parece evidente la necesidad de ampliar con un Segundo Ciclo, la Diplomatura de Relaciones Laborales. Sin entrar en cuestiones de otro tipo y siguiendo en la perspectiva de los contenidos, sencillamente porque es imposible en tres años formar a un experto, a un auténtico especialista en el mundo de las relaciones laborales.

${ }^{3}$ GONZÁLEZ ORTEGA, S.: Valoración de los actuales estudios de Relaciones Laborales y propuesta de un eventual ciclo para dichas enseñanzas. (1995). 
Desde el punto de vĩsta de la gestión, el debate se centró en las dificultades que plantean las "asignaturas llave", convalidaciones, asignaturas de múltiple asignación,libre configuración y, muy especialmente, sobre las prácticas integradas. En primer lugar convendría aclarar qué entendemos por prácticas integradas. Aunque en el espíritu del decreto se puede apreciar una intención por proporcionar a los alumnos la posibilidad de que confronten y complementen sus conocimientos teóricos con el mundo laboral, con la realidad que van a encontrar cuando acaben sus estudios, no aporta las elementos necesarios para resolverlo en este sentido. A saber, quíen sería en este caso el encargado de buscar las plazas para los alumnos en prácticas -el propio centro, un servicio de la universidad que atiende a todas las titulaciones y que no en todas las universidades existe. Si se gestionan desde el propio centro es necesario contar con mas medios. Al mismo tiempo los departamentos se tienen que hacer cargo de que el seguimiento de dichas practicas no se puede hacer desde los despachos, sino que requieren un contacto frecuente con las empresas e instituciones donde los alumnos están completando su formación. En este campo como en otros que hemos ido mencionando, hablamos de una nueva concepción de nuestros estudios. En este sentido nos tropezamos con frecuencia, con empresarios y jefes de personal que aún no conocen las posibilidades de nuestros alumnos, se impone por lo tanto, en primer lugar una labor de información y de convencimiento, de que efectivamente, pueden realizar trabajos de cierta envergadura. En este trabajo de información contamos con la dificultad de la existencia de una gama, mas o menos amplia -según las comunidades autónomas- de programas de prácticas: unas remuneradas, otras no, unas a realizar exclusivamente durante el verano, otras a lo largo del curso, unas gestionadas desde los vicerrectorados de relaciones externas o de extensión universitaria, otras desde los sindicatos, otras desde los propios centros, etc.

También podemos entender que la integración a la que alude el decreto, es una integración entre las áreas a las que se asignan las mencionadas prácticas. Por lo tanto, las tres áreas en cuestión tendrían que coordinar un programa de prácticas internas.

Con imaginación y cuando se puede, los centros están intentando ofrecer al mismo tiempo prácticas internas integradas y prácticas externas. $\neq$ La diversidad de los modelos utilizados es muy considerable, dependiendo de las disponibilidades de recursos humanos apropiados en los distintos centros, de las condiciones empresariales y laborales de la propia ciudad, etc. En este campo nos encontramos en muchas ocasiones y de un modo tenaz contra las 
trabas administrativas que impone la propia universidad. La nueva situacion exige adaptarnos al ritmo de funcionamiento de la empresa privada y superar obstáculos en la administración pública.

\section{Conclusiones.}

1.- La necesidad de celebrar de forma periódica este tipo de encuentros para seguir discutiendo, intercambiando opiniones, incluso programas, exponiendo posibles soluciones, etc. sobre las múltiples y nuevas situaciones que se están planteando. En este sentido es de elogiar las buenas relaciones que se mantienen entre las distintas escuelas y la ágil articulación en su manera de funcionar que posibilita la existencia de una comision permanente de directores a nivel nacional ${ }^{4}$.

2.- Evitar el excesivo protagonismo de alguna de las áreas sobre el resto, atendiendo a la necesaria interdisciplinariedad de la titulación. Respetando el mínimo necesario a las áreas que sin ser de gran peso tienen que estar presentes.

3.- Necesidad imperiosa de que los departamentos se impliquen de una manera eficaz en el desarrollo de los nuevos planes. Lo hicieron con ahínco en el momento de su elaboración, han de acerlo ahora en su puesta en práctica.

4.- Se hace necesario un mayor apoyo institucional, en cuanto a recursos económicos, humanos, favorecer convenios con empresas e instituciones, agilizar la gestión,etc.

A título particular me atrevo a apuntar un problema de mentalización; es decir, nos enfrentamos a un nuevo plan de estudios, que los alumnos, no tienen argumentos para rechazar a priori, puesto que no lo conocen, aunque -eso sí- sean tremendamente receptivos a las noticias sobre su mal funcionamiento en términos generales. Es necesario, a mi juicio, llevar a cabo una labor de concienciación en los dos sectores directamente implicados: por un lado y fundamental los profesores, y por otro los alumnos. Entre todos nos

- En el Pleno de directores celebrado en la última sesión de las Jornadas y a propuesta de la Escuela de Salamanca, se acordó que las próximas tuvieran lugar en dicha ciudad, y en fechas que serán oportunamente comunicadas. 
tenemos que convencer de que este nuevo sistema requiere una mentalización diferente, un ritmo de trabajo distinto. Es cierto que tiene fallos y acabamos de enumerar los más claros, pero, a mi juicio, no son determinantes, no impiden que la formación sea mucho mejor, que la adecuación a las demandas laborales sea más alta. Ni mucho menos. Los nuevos planes de estudios tienen ventajas comparativas muy importantes:

a) mejor formación

b) se le van incorporando nuevos perfiles laborales a medida que los recién diplomados se van incorporando al mercado laboral

c) permiten, a traves de las optativas, elaborar diferentes itinerarios curriculares.

Y sobre todo, los fallos están localizados y se puden subsanar, es necesario eso sí, que exista un mínimo de voluntad en las partes implicadas. En los alumnos que estén dispuestos a marcarse un nuevo y mas intenso ritmo de trabajo, y en los profesores, que le dediquen a la docencia y a la adaptación a estos nuevos planes todo el esfuerzo necesario, prescindiendo de intereses departamentales en función del objetivo último que es la formación de buenos profesionales .

Es cierto que muchos de los problemas aquí señalados son imputables a la estructura y al funcionamiento del conjunto de la universidad española, pero no debe ser excusa para abandonar y hacer de esta diplomatura una carrera vacía y alejada del fin para el que fue concebida, sino todo lo contrario. Desde los centros existe un claro convencimiento de que efectivamente tiene un margen importante en el mercado de trabajo, si entre todos somos capaces de llenar de contenido útil las asignaturas que integran su plan de estudios. 